\title{
Humic acid promoted goethite bioreduction, hydroxyl radical production and sulfanilamide degradation during anaerobic- aerobic transition
}

\author{
GUANGFEI LIU ${ }^{1 * A N D}$ HuALI YU ${ }^{1}$ \\ ${ }^{1}$ School of Environmental Science and Technology, Dalian \\ University of Technology, Dalian 116024, China \\ (*correspondence: guangfeiliu@dlut.edu.cn)
}

The biogeochemical cycles of $\mathrm{Fe}$ and $\mathrm{C}$ are always intimately associated in natural environments. It has been found that abundant hydroxyl radical $(\cdot \mathrm{OH})$ was produced during the oxygenation of reduced Fe-bearing minerals at anaerobicaerobic interface. However, little was known about the impacts of coexisting natural organic carbon on this process. Here, it was found that $10-100 \mathrm{mg} / \mathrm{L}$ humic acid (HA) could significantly promote the reduction of both abiogenic and biogenic goethite $\left(\mathrm{Gt}_{\mathrm{chem}}\right.$ and $\left.\mathrm{Gt}_{\mathrm{bio}}\right)$ by Shewanella oneidensis MR-1 under anaerobic condition. When trasferred to aerobic environment, systems containing higher Fe(II) concentrations generated higher concentrations of $\bullet \mathrm{OH}$, whereas the production of $\cdot \mathrm{OH}$ by oxidation of reduced HA in these systems was negligible. Adsorbed Fe(II) played a significant role in $\cdot \mathrm{OH}$ generation, and contributed to at least $72.8 \%$ and $90.1 \%$ of $\cdot \mathrm{OH}$ generation in the systems of $\mathrm{Gt}_{\mathrm{chem}}+\mathrm{HA}$ and $\mathrm{Gt}_{\text {bio }}+\mathrm{HA}$. HA-complexed $\mathrm{Fe}$ (II) contributed to the rest $\bullet \mathrm{OH}$ generation. Sulfanilamide (SA), a widespread antibiotics was selected as typical pollutants to assay the degradation performance of different systems. The trend of SA degradation was consistent with that of $\bullet \mathrm{OH}$ generation. About $15.2 \%-72.5 \%$ of SA was degraded in different systems. Four redox fluctuation cycles with different regimes were condcuted to evaluate the sustainability of $\bullet \mathrm{OH}$ production in subsurface environments with different redox conditions. Stable production of $\bullet \mathrm{OH}$ were obtained in repeated cycles with "fluctuation 3:1" (3-day anaerobic and 1-day aerobic), while $\cdot \mathrm{OH}$ production decreased with the increasing cycles of "fluctuation 6:1" (6-day anaerobic and 1-day aerobic), suggesting that higher fluctuation frequency might facilitate the stable $\cdot \mathrm{OH}$ production. While the crystallinity of both $\mathrm{Gt}_{\text {chem }}$ and $\mathrm{Gt}_{\text {bio }}$ decreased with increasing anaerobic-aerobic transitions, the repeated redox fluctuation did not cause the formation of new crystalline mineral phase. In conclusion, our findings indicated that the presence of $\mathrm{HA}$ promoted $\bullet \mathrm{OH}$ generation and oxidative contaminant degradation by enhancing the production of adsorbed and complexed Fe(II) under redox fluctuation conditions. 